\title{
Comparative evaluation of automated reading versus visual interpretation with the BBL Crystal enteric/nonfermenter identification system in a clinical setting
}

\author{
J. R. Lo-Ten-Foe • M. A. C. Ververs • A. G. M. Buiting
}

Published online: 23 May 2007

(C) Springer-Verlag 2007

The BBL Crystal enteric/nonfermenter identification system (Becton Dickinson, Sparks, MD, USA) is a miniaturized commercial system that is widely used to identify more than 500 gram-negative bacilli [1-4]. The system is designed to identify members of the family Enterobacteriaceae as well as common isolates of clinically significant glucosenonfermenting gram-negative bacilli. Many of the tests used in this system are modifications of classical methods. Included among them are tests for fermentation, oxidation, degradation and hydrolysis of various substrates. In addition, there are chromogen-linked substrates to detect enzymes used by microbes to metabolize various substrates. The reaction patterns are converted into a ten-digit profile number. In addition, indole and oxidase tests are performed. The reaction patterns for the substrates of a wide variety of gram-negative bacilli are stored in the BBL Crystal identification database. Identification is achieved by comparing the substrate reaction patterns of the test isolate with those stored in the database.

The reaction patterns for the substrates can be interpreted visually, using the Crystal light box and color chart, or with the BBL Crystal autoreader (Becton Dickinson). Two calculations are made - biotype validity and a confidence value. The BBL Crystal autoreader is designed to read Crystal miniaturized systems and subsequently transfer the automated readings to a personal computer for identification with the database. The advantage of using the BBL Crystal autoreader is it eliminates subjective human interpretation of the readings; in addition, it increases speed and

J. R. Lo-Ten-Foe $(\bowtie) \cdot$ M. A. C. Ververs · A. G. M. Buiting Division of Medical Microbiology,

St. Elisabeth Ziekenhuis Tilburg,

Hilvarenbeeksweg 60,

5000 AS Tilburg, The Netherlands

e-mail: j.lotenfoe@elisabeth.nl eases execution. The BBL Crystal enteric/nonfermenter ID system (Becton Dickinson) is generally accepted as a reliable method for identifying gram-negative bacilli [5-7]. The objective of our study was to use clinical isolates to test whether the BBL Crystal autoreader generates consistent results compared to manual reading of the substrate reaction patterns.

A total of nine ATCC reference strains were included as quality controls (ATCC17925 Acinetobacter lwoffii, ATCC5030 Enterobacter cloacae, ATCC25922 Escherichia coli, ATCC33495 Klebsiella pneumoniae, ATCC8427 Proteus vulgaris, ATCC35032 Pseudomonas aeruginosa, ATCC13525 Pseudomonas fluorescens, ATCC 49128 Pseudomonas putida, ATCC13637 Stenotrophomonas maltophilia). These ATCC reference strains were correctly identified using the BBL Crystal enteric/nonfermenter identification system in combination with either visual interpretation or utilization of the BBL Crystal autoreader. Visual interpretation was performed separately by three experienced laboratory technicians.

In total, we tested 200 gram-negative bacillus strains isolated from urine $(n=80)$, sputum $(n=55)$, blood $(n=20)$ or tissue samples $(n=45)$ according to the manufacturer's instructions. We subsequently interpreted the results both visually and automatically using the BBL Crystal autoreader. The isolates were considered representative of those commonly encountered in the clinical laboratory and included 150 Enterobacteriaceae and 50 glucose nonfermenting bacilli. The gram-negative bacilli we included were independent isolates that were recently obtained from clinical specimens processed in the medical microbiology laboratory of our hospital. These clinical isolates included K. pneumoniae $(n=16)$, Klebsiella oxytoca $(n=10)$, Salmonella spp. $(n=12)$, Shigella spp. $(n=3)$, Shigella sonnei $(n=2)$, E. coli $(n=19)$, Enterobacter aerogenes 
$(n=12)$, Enterobacter cancerogenus $(n=1)$, E. cloacae $(n=$ 12), P. vulgaris $(n=5)$, Proteus penneri $(n=1)$, Proteus mirabilis $(n=12)$, Serratia marcescens $(n=10)$, Serratia liquefaciens $(n=1)$, Serratia odorifera $(n=1)$, Citrobacter freundii $(n=5)$, Citrobacter amalonaticus $(n=2)$, Citrobacter koseri $(n=5)$, Yersinia enterocolitica $(n=2)$, Hafnia alvei $(n=4)$, Providencia rettgeri $(n=1)$, Providencia alcalifaciens $(n=1)$, Providencia rustigiani $(n=1)$, Morganella morganii $(n=8)$, Aeromonas hydrophila $(n=1)$, Pasteurella multocida $(n=3)$. Glucose nonfermenting clinical isolates included P. aeruginosa $(n=18)$, P putida $(n=1)$, Pseudomonas stutzeri $(n=1)$, S. maltophilia $(n=12)$, A. lwoffii $(n=5)$, Sphingomonas paucimobilis $(n=1)$, Acinectobacter baumannii $(n=9)$, Plesiomonas shigelloides $(n=1)$, Chryseobacterium indologenes $(n=2)$. Discrepancies between the methods of interpretation were resolved by confirming the identity of the clinical strains using phenotypic testing methods or $16 \mathrm{~S}$ rDNA sequencing as described by Vaneechoutte et al. [8].

Discrepancies were noted in three isolates. One P. putida strain was visually interpreted as $P$. stutzeri (confidence value 0.4916 ) or $P$. fluorescens (confidence value 0.3406 ), while the BBL Crystal autoreader identified it as $P$. aeruginosa (confidence value 0.4612), P. putida (confidence value 0.3034 ), or $P$. fluorescens (confidence value 0.2022 ). Neither method of interpretation resulted in correct identification to the species level, indicating the BBL Crystal enteric/nonfermenter ID system has a limited ability to correctly identify this strain. In the second case an E. coli strain was identified correctly with visual interpretation, but interpretation using the BBL Crystal autoreader resulted in an unacceptable profile. Retesting the sample with the BBL Crystal enteric/nonfermenter identification system followed by both methods of interpretation resulted in an unacceptable profile with visual interpretation and a correct identification in the BBL Crystal autoreader. In the third case, a $H$. alvei strain yielded an unacceptable profile with both methods of interpretation. If we omit the first and third cases, considering them reflections of the limitation of the BBL identification Crystal enteric/nonfermenter identification system itself rather than a failure of the two interpre- tation methods, we find a concordance of $99.5 \%$ between the BBL Crystal autoreader and visual interpretation. Investigation of the differences in the ten-digit codes generated by either visual interpretation or the BBL Crystal autoreader, revealed no obvious bias in either method.

Based on these data, we conclude that the BBL Crystal autoreader performs well as an alternative to the visual interpretation of the BBL Crystal reaction patterns in a clinical setting. The major advantages of this method are its increased speed and the elimination of subjective human interpretation of the readings. The BBL Crystal autoreader would be a useful addition to the microbiology laboratories of most hospitals.

\section{References}

1. Holmes B, Costas M, Thaker T, Stevens M (1994) Evaluation of two BBL Crystal systems for identification of some clinically important gram-negative bacteria. J Clin Microbiol 32:2221-2224

2. Wauters G, Boel A, Voorn GP, Verhaegen J, Meunier F, Janssens M, Verbist L (1995) Evaluation of a new identification, Crystal enteric/ non-fermenter, for gram-negative bacilli. J Clin Microbiol 33:845-849

3. Robinson A, McCarter YS, Tetreault J (1995) Comparison of Crystal enteric/nonfermenter system, API20E, and Vitek automicrobic system for identification of gram-negative bacilli. J Clin Microbiol 33:364-370

4. Peele D, Bradfield J, Pryor W, Vore S (1997) Comparison of identifications of human and animal source gram-negative bacteria by API 20E and Crystal E/NF systems. J Clin Microbiol 35:213216

5. Micklewright IJ, Sartory DP (1995) Evaluation of the BBL Crystal enteric/nonfermenter kit for the identification of water-derived environmental Enterobacteriaceae. Lett Appl Microbiol 21:160-163

6. Soler L, Marco F, Vila J, Chacon MR, Guarro J, Figueras MJ (2003) Evaluation of two miniaturized systems, MicroScan W/A and BBL Crystal E/NF, for identification of clinical isolates of Aeromonas spp. J Clin Microbiol 41:5732-5734

7. Varettas K, Mukerjee C, Schmidt M (1995) A comparative study of the BBL Crystal enteric/nonfermenter identification system and the bioMérieux API20E and API20NE identification systems after overnight incubation. Pathology 27:358-361

8. Vaneechoutte M, Claeys G, Steyaert S, De Baere T, Peleman R, Verschraegen G (2000) Isolation of Moraxella canis from an ulcerated metastatic lymph node. J Clin Microbiol 38:3870-3871 